\title{
Web-Oriented Architecture (WOA) Enabled Customer-Centric Collaborative Commerce Platform (WCCP)
}

\author{
Chuan-Jun Su
}

\begin{abstract}
Traditional Service-Oriented Architecture (SOA) has consistently promised more than it has delivered, but Web-Oriented Architecture (WOA) promises to deliver the robust outcomes formerly expected of SOA. WOA extends SOA to web-based applications. The architecture hides the protocol complexity in the application state and provides simpler methods for the deployment of interoperable web services. However, this pragmatic and effective approach has only been rarely implemented, limiting the body of formal knowledge and support available to enterprises which want to exploit this profoundly useful and simple way of developing interoperable systems.

In this paper, we describe the development of a novel customer-centric collaborative commerce platform (WCCP) on WOA in a virtual environment. The classification from the United Nations Standard Products and Services Code (USNPSC) was adopted to achieve the interoperability between differential enterprises.
\end{abstract}

Index Terms-Collaborative commerce, SOA, UNSPSC, WOA.

\section{INTRODUCTION}

The global business environment is rapidly evolving. Competition is fiercer and customers are more demanding. Product life cycles are shorter and time to market has accelerated. Enterprises and distributers are anxious to have a comprehensive product and service market but they usually cannot focus on their core competitive products. These challenges require enterprises to collaborate effectively and share resources with upstream suppliers, partners, and downstream customers.

Collaborative commerce (C-Commerce) can be defined as "an application of an interorganizational information system for electronic collaboration between business partners and organizational employees" [1]. C-Commerce is an emerging trend for improving the efficiency of sales, procurement, manufacturing, distribution, replenishment, and other activities, which uses information technology as backbone to achieve a closer integration and stronger relationships between these activities [2].

Even though Service Oriented Architecture (SOA) initiatives around the world target the right goals, most efforts have fallen profoundly short of desired levels of integration and improved business agility [3]. SOA implementation involves complex Web Service standards

Manuscript received May 14, 2014; revised July 30, 2014.

Chuan-Jun $\mathrm{Su}$ is with the Department of Industrial Engineering \& Management, Yuan Ze University, Taiwan, R. O. C. (e-mail: iecjsu@saturn.yzu.edu.tw). such as SOAP, UDDI, WSDL, etc. introduced by IBM and Microsoft, and some reports [4] show rather disappointing results regarding return on investment (ROI) and business alignment. Despite SOA failing in the vast majority of integration scenarios, it continues to be prevalent in most enterprises. Enterprises need simpler methods to achieve the benefits promised by SOA. A bottom-up web-oriented architecture (WOA) approach, developed through grassroots movements and based on web 2.0 resources, seems to offer a better route to the benefits of SOA.

WOA merges the core design of SOA with Web 2.0-style user generated content and mashups, and regards entire web as native resources. This new subset of SOA adapts the concept of Representational State Transfer (REST) to represent resources located within enterprises with Uniform Resource Identifiers (URIs). Users can access and interoperate resources on heterogeneous platforms via simple URIs. WOA regards the Internet as a global SOA: every website, web service or web page is a resource to be shared, integrated, and reused to create new functions on the Internet in way that is both flexible and interoperable. These newly created functions can not only be accessed independently but can also be treated as resources or items in this global SOA and then can be separated, integrated and reused to create still other functions. WOA is a style of software architecture that extends service-oriented architecture (SOA) to web-based applications, and is sometimes considered to be a light-weight version of SOA.WOA aims at maximizing browser and server interactions through technologies such as REST [5] and Plain Old XML (POX) [6].

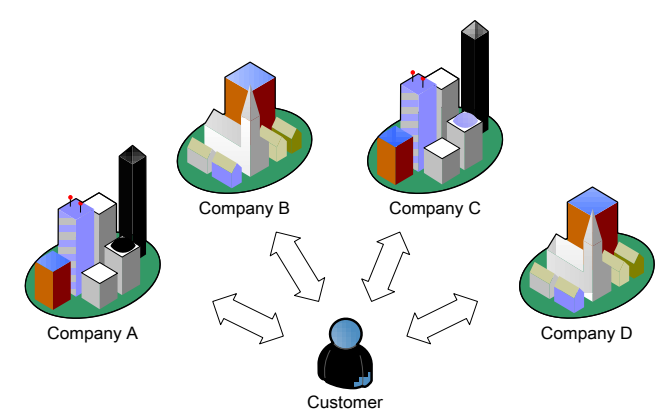

Fig. 1. Traditional e-commerce.

In the traditional e-commerce environment, each company has their own online commerce platform. Companies are anxious to provide a comprehensive product and service market and satisfy the demands of customer, even though most selling are the core products and services. Hence, in order to provide a comprehensive market companies usually cannot focus on their core competitive products and services. 
Relatively, when a customer needs products and services from different companies, he/she should go to different platform to fulfil his/her needs as shown in Fig. 1.

In C-commerce, designated individuals and groups within an enterprise and including its suppliers, customers, and partners, work together for mutual gain. Customers are empowered to select different kind of products and services from various companies according to their personal preferences while companies are able to focus on their core competencies as shown in Fig. 2.

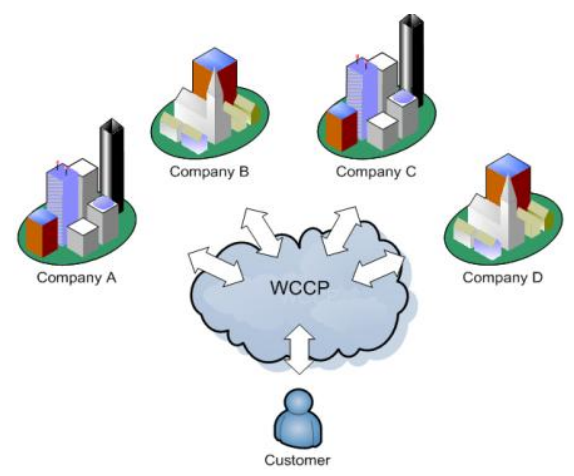

Fig. 2. WCCP-supported C-commerce.

To achieve C-commerce, online commerce marketplaces require an interoperable environment for products and services representations among suppliers, customers, and partners. Voiding the manual creating problems, for instance, a large number of concepts specifications and the time of product innovation maintenance, the United Nation Standard for Product and Service Code (UNSPSC) [7] is an applicable approach. Therefore, through the sharing product information across this collaborative commerce process, enterprises and business partners can improve the efficiency of business process integration and heterogeneity of the different business model.

This paper aims to demonstrate WOA's potential as a simpler method for developing a customer-centric collaborative commerce platform (WCCP), incorporating Ajax technology in a 3D setting. With WCCP and WOA, manufacturing resources, products or services (represented by the REST concept) can be interoperated across heterogeneous platforms via simple URIs. Using UNSPSC classification to integrates the terminology of products and services among different collaborative partners. Therefore, resources, products and services can be integrated and reused to create new functions or services on the WCCP. In addition, the WCCP allows resources, products and services run in a 3D environment, enabling developers to provide friendlier and more intuitive user interfaces (UIs). In the system development section and scenarios, this paper presents a collaborative work on furniture selling as an example to validate the viability and feasibility of the proposed approach.

\section{RELATED WORKS}

\section{A. Web-Oriented Architecture (WOA)}

WOA is a simpler subset of SOA and can be thought of as "WOA = SOA + WWW + REST". WOA was first proposed by Nick Gall [8], who indicated that there are many ways to implement SOA. WOA essentially describes a core set of web protocols like HTTP and plain XML as the most dynamic, scalable and interoperable web service approach. Instead of complex standards in conventional SOA, WOA advocates REST, an increasingly popular, powerful, and simple method of leveraging HTTP as a Web service, initially proposed by Roy T. Fielding [9]. Enterprises usually do not need anything more complex than HTTP, and they can efficiently build up or share their own services over the Internet with REST and WOA. Their customers or Internet surfers can remix those services to create new functions.

Dion Hinchcliffe pointed out the basic tenets of WOA and some significant contrasts between WOA and SOA [10]. Roger Smith [4] indicated that WOA and SOA are complementary architectural styles of differing levels of sophistication. While WOA may serve the purposes of some implementations, others would require scaling up to full SOA. Recently, advances in a new generation of XML hardware appliances has begun to refute the argument that WOA is compelling for simple ad hoc development but that companies need to stick to the full Web services SOAP stack if they want secure, reliable, manageable services. These devices from suppliers including Cast Iron Systems, Cisco, IBM DataPower, and Sonoa Systems provide fast and secure routing of XML, and provide interoperability among various WOA/SOA/Web 2.0/software-as-a-service preferences of corporate customers and their partners. To that extent, existing SOAs can be extended with resource endpoints, and new WOAs can be extended with service-oriented end points. In many cases, hybrid systems combining both WOA and SOA features may make the most sense. WOA is not suitable for all applications, and high-end enterprise applications in particular require the more sophisticated portions of the SOA stack. For the vast majority of uses, however, WOA is the most interoperable, easiest to implement, and most highly scalable technique for building practical and open Web services that anyone can use.

\section{B. UNSPSC}

It becomes important to classify products and services with a common coding scheme between upstream suppliers, partners, and downstream customers. Consequently, it requires a single coding convention that all partners could follow. The United Nations Standard Products and Services Code (UNSPSC) [7] is an open, standardize and hierarchical product and service classification. It is used extensively around the world in various online commerce marketplaces. UNSPSC is a five-level hierarchical classification. An extracted example is shown in Table I.

TABLE I: AN EXAMPLE OF UNSPSC

\begin{tabular}{|c|c|c|}
\hline UNSPSC & Number & Title \\
\hline Segment & 56 & Furniture and Furnishings \\
\hline Family & 10 & Accommodation furniture \\
\hline Class & 15 & Furniture \\
\hline Commodity & 02 & Sofas \\
\hline Business Function & 14 & Retail \\
\hline
\end{tabular}

\section{THE WCCP FRAMEWORK}

A RESTful web service (also called a "RESTful web API") 
is a simple web service implemented using HTTP and the principles of REST. Such a web service can be thought of as a collection of resources. The WOA-based WCCP framework comprises 1) a Data Access Layer which stores backend data of the platform and the information from suppliers, 2) RESTful web services, and 3) a User Interface Layer which renders the front-end user interfaces (UIs) for each participant. RESTful web services enable suppliers to publish their core products and services in the WCCP with simple URIs and render these published products and services interoperable in the UNSPSC classification.

Through the Internet, users are allowed to independently access these services through HTTP methods such as POST, GET, PUT and DELETE; and to integrate and reuse them to create new services. Through the WCCP, users can gather and execute various applications and services from other web resources, third-party mashup tools, and firms. The WCCP also provides a mashup function to combine registered services into new services. The multi-layer WCCP framework comprises a Data Access Layer, a RESTful web service, and a User Interface Layer as illustrated in Fig. 3:

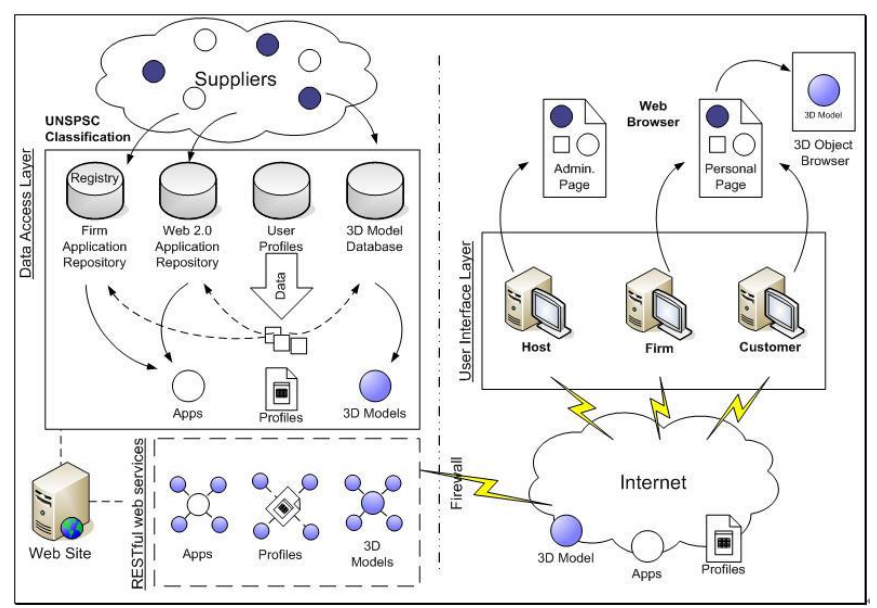

Fig. 3. The framework of WCCP.

\section{A. Data Access Layer (DAL)}

The DAL is responsible for storing static data (e.g., User Profiles, 3D models) and applications furnished by firms and Web 2.0 communities through WCCP APIs. To achieve interoperability, suppliers are required to conform the description of products and services to the UNSPSC classification. DAL encompasses four storage areas: 1) Firm Applications Repository, 2) Web 2.0 Applications Repository, 3) 3D Model Database, and 4) User Profiles:

- Firm Apps Repository - stores all registered resources or indexes from firms. Firms can focus on their core competencies in WCCP and publish their web services through REST. Registered users may then access these services or create new services by using third-party mashup tools or the mashup functions provided by the WCCP.

- Web 2.0 Apps Repository - a database that stores all services created by users or firms. The services are usually created by users using mashup tools or created by users and/or firms remixing the firms' services with WCCP's APIs.

- 3D Model DB - all 3D product models running on the platform are stored in here. These models are originally created by the Host or uploaded by users or firms.

- User Profiles: stores the participators' profiles that encapsulate personal information and configuration, the list of uploading applications or models, and the interrelationships between 3D models and applications.

\section{B. RESTful Web Service}

WOA itself strongly opts to the simplest, most open, Web-scaled technical approaches. An important aspect of WOA is to use REST to hide the complexity of SOA and to provide interoperability with other applications through simple URIs. The resources in REST are referenced with a global identifier (e.g., a URI in HTTP). To manipulate these resources, components of the network communicate via a standard interface (e.g., HTTP) and exchange representations of these resources. All resources transfer their state between client and resource through a uniform interface, encompassing HTTP methods POST, GET, PUT and DELETE. These methods are often respectively associated with the database methods CREATE, READ, UPDATE, DELETE (CRUD). RESTful web services in the WCCP provide an abstraction for publishing information and giving remote access to the Data Access Layer. They regard the contents of the Data Access Layer as resources, each of which is referenced with a simple URI.

\section{User Interface Layer (UIL)}

The UIL renders the front-end user interfaces based on a user's role. It encompasses two major views: 1) Personal Page and 2) Administration Page. When users or firms log in to the WCCP, the Personal Page would be invoked to present a virtual environment, related services and personal information. For example, in the UIL users can configure or maintain personal virtual rooms, decorate rooms with 3D furniture/objects, transform 3D objects, and relate each 3D object to a service which they are interested in. This page contains an Extensible 3D (X3D) plug-in to enable 3D objects viewing. The Administration Page allows hosts to manage users' profiles, applications or services, and 3D models in WCCP.

\section{IMPLEMENTATION}

The WCCP prototype was successfully implemented and tested on IE $^{\mathrm{TM}}$ and FireFox ${ }^{\mathrm{TM}}$ with Ajax support. The JavaScript library JQuery was utilized for developing WCCP functions. The Vivaty ${ }^{\mathrm{TM}}$ X3D Player was used to render X3D-constructed 3D scenes.

JSP (Java Server Page) was adopted as the CGI (Common Gateway Interface) middleware and Apache Tomcat v 5.5 as the web container running under J2SDK 1.6 for communication between front-end web pages and back-end web server. We used Microsoft ${ }^{\mathrm{TM}}$ Access 2003 with Java Database Connectivity (JDBC) to implement the Data Store. Finally, we used Open Database Connectivity (ODBC) as a driver to connect applications and the Data Store.

RESTful Web Service is a simple web service that can be thought as a collection of resources implemented using HTTP and the principles of REST. Various technologies and 
frameworks can be used to develop RESTful Web Services, including Ruby On Rails (ROR) [11], Restlet [12], Cetia4 [13], etc. In WCCP, a Java Servlet-based REST Framework for Web Service Applications called Cetia4 ${ }^{\mathrm{TM}}$ was adopted for developing REST web service applications that answer HTTP stateless requests.

\section{USAGE SCENARIOS AND DEMONSTRATION}

Thanks to the new generation of Web 2.0, more and more new business model existed. The demands of the company's customers are growing more diverse, and the company needs to adopt more agile approaches, allowing it to respond more quickly to changing customer tastes. ABC Company is one of the new rising companies decided to launch eHouse a virtual store that allows customers to pick up furniture and custom-design their house, supported by a WCCP-based system which enables the company and its suppliers to collaborate to fill custom orders.

eHouse allows the company to focus on its core competencies, resulting in reduced production costs and response times [14]. Consumers, as innovators, are enabled to freely "design" their house in a 3D environment, seamlessly pick up furniture products from different suppliers. Each 3D furniture product encapsulates information including the supplier's profile and availability, etc. All 3D objects and functions can be accessed through HTTP methods GET and POST with a simple URI. These functions are regarded as resources on the Internet, which may be reused by the user to create customized functions. This collaborative commerce model not only allows greater variation in the finished demand, but also allows the customers to further compare their products among different suppliers.

\section{A. User Scenario 1: Supplier's Perspective}

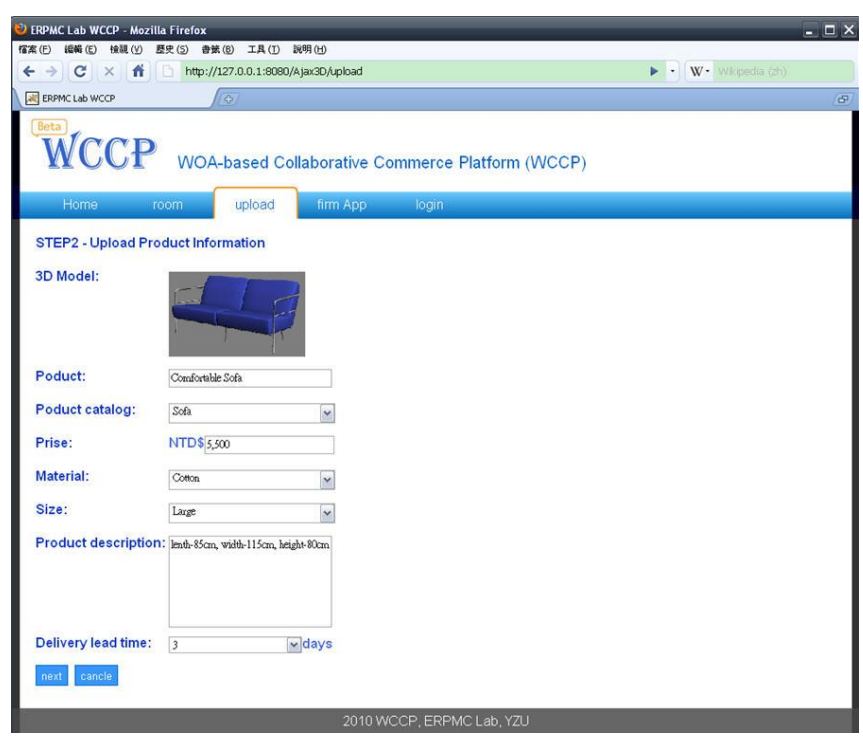

Fig. 4. Uploading information to WCCP.

MakingSofa, Inc. is a furniture manufacture company specializes in producing sofas. The company joined eHouse so it could focus more on its core competencies and to reduce market mediation costs, production costs, and turnaround times. After registration, the company publishes its product data (3D models), company profile, and delivery lead times as depicted Fig. 4. The published data are intrinsically stored as resources in eHouse's Firm Applications Repository as a registered service and can be accessed through simple URIs, for example, http://MakingHandle.com/[userid]/MakingSofa to access the company's information.

Other sofa suppliers also register and publish their services with eHouse. These services, coming from suppliers producing the same components, can be mashed up to create a new service called SearchSofaSupplier through WCCP's built-in mashup function as illustrated in Fig. 5. The new service SearchSofaSupplier is stored in the Web 2.0 Application Repository.

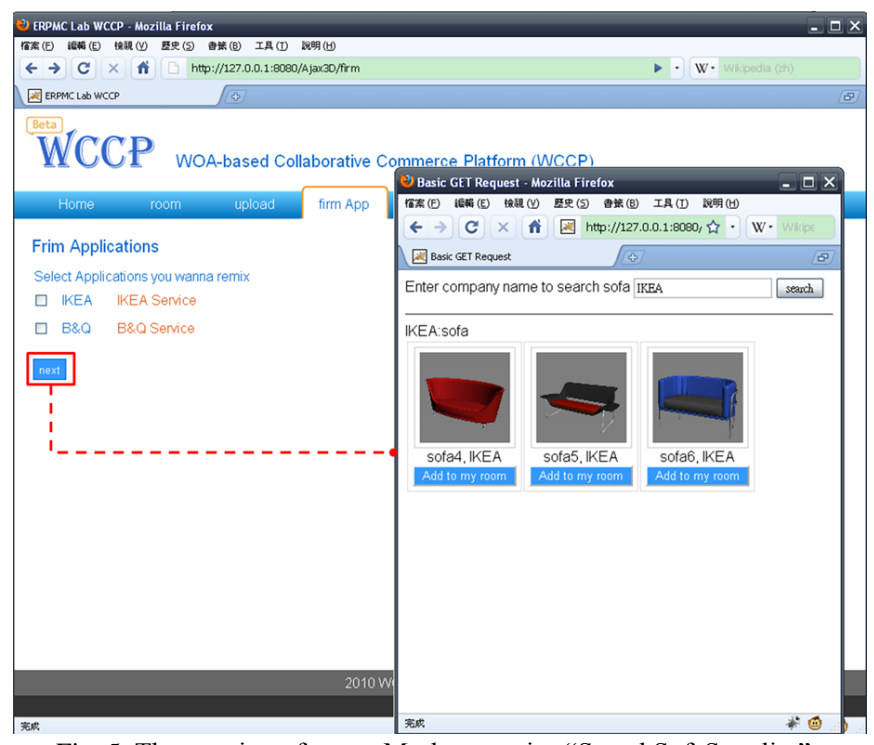

Fig. 5. The creation of a new Mashup service "SearchSofaSupplier".

\section{B. User Scenario 2: Consumer's Perspective}

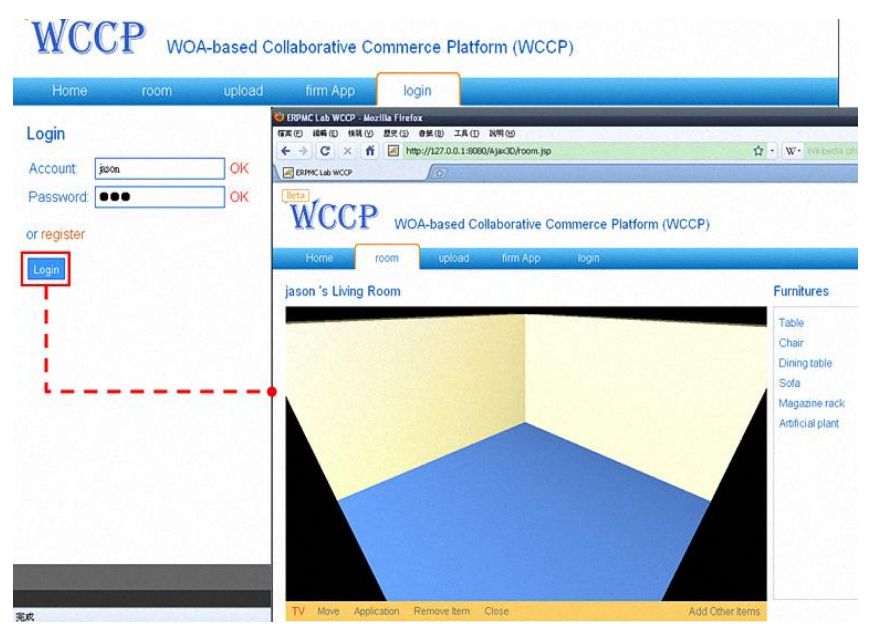

Fig. 6. User logs into the WCCP and views the default room in 3D.

Jason is a newly rich engineer just bought a new house in Taiwan. He want to buy some furniture especially a fancy and suitable sofa so that he can feel relax and comfortable when he back home. He registers on eHouse and finds a default 3D living room ready for decoration. He chooses sofa, table and chair from the application repository that stores product models from various suppliers, and begins decorating his living room as illustrated in Fig. 6 and 7. All 
3D models are interrelated with services or applications stored in the Web 2.0 Application Repository or Firm Application Repository. When Jason clicks on the sofa in the 3D mockup, the SupplierInfo service shows inventory availability and other supplier-related information as shown in Fig. 8. When the decoration is complete, the system displays order details including expected delivery lead time, shipping, and billing information. Lastly, the respective suppliers will be informed to ship all the furniture to Jason's new house.

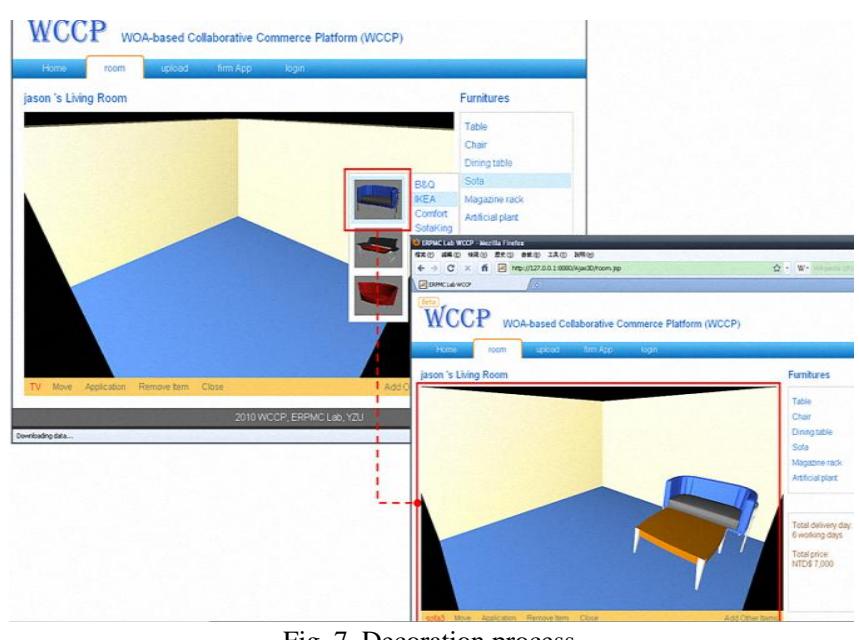

Fig. 7. Decoration process.

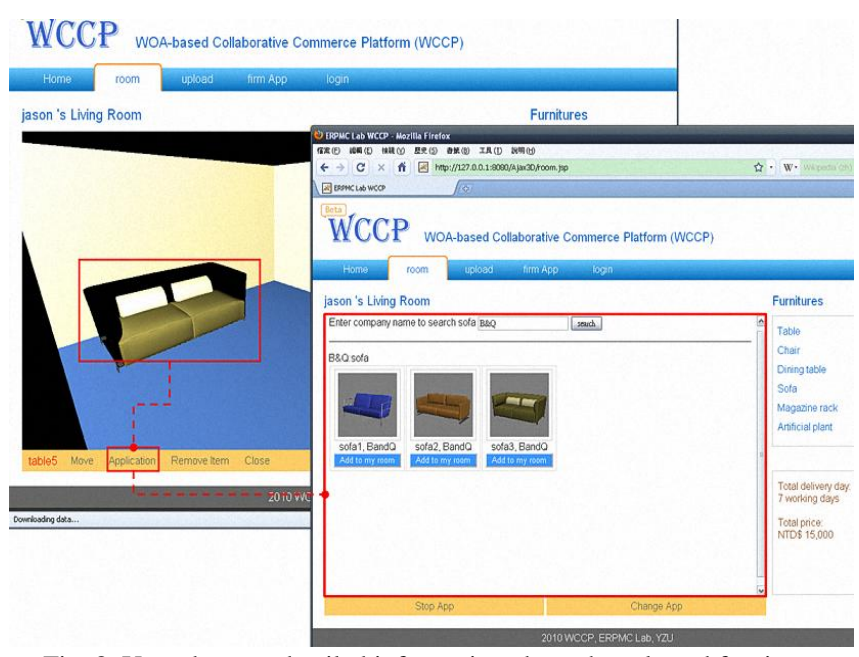

Fig. 8. Users browse detailed information about the selected furniture.

\section{CONCLUSION AND FUTURE WORKS}

\section{A. Conclusion}

Facing unpredictable and rapidly-changing markets, enterprises are increasingly looking to Collaborative Commerce for solutions. These collaborative works require effective communication, interoperation and integration at the resource level. While SOA provides a robust architecture for interoperability between these heterogeneous platforms, implementation requires, CIOs and system architects to deal with complex WS-* standards, often leading to poor results and disappointing ROI.

Enterprises need simpler methods to achieve the benefits of SOA. WOA, which merges the core design of SOA, Web 2.0 resource sharing, and mashup functions, seems to offer a solution. It treats the Internet as a global SOA with every website, web service, or web page a usable resource or item. Users can share, integrate, and reuse all of these resources to create new functionalities on the Internet in a loosely-coupled and interoperable way. These new functionalities can not only be accessed directly, but can also be treated as resources or items of this global SOA, and thus be integrated and reused to create other functionalities.

At the same time, the semantic concept of UNSPSC classification provides an effective approach of integrating various business parties. Company can via UNSPSC uses the same classification to describe the products and services.

In this paper, we have described a WOA-based and approach to creating integrated and interoperable systems and have used the WCCP open platform to create a WOA-based collaborative environment in Collaborative Commerce. Simultaneously, we developed a house furniture decoration environment as an example. The Host organizes a collaborative team and builds up the WCCP based on their demands. The REST concept allows users to independently access and reuses each function through a simple URI. The manufacturer also benefits from analyzing user-generated specs which offer insight into market demands. The WCCP framework also offers firms an environment for their APIs or internal data through REST, but also enables them to take advantage of mashups with other firms' applications to create new services and functions, thus adding value to their own applications. Once a firm has established its applications through WOA it may, depending on its own requirements, upgrade to full SOA or continue with the WOA deployment. Furthermore, WCCP provides advantages to the user in that the $3 \mathrm{D}$ rendition of the room provides an intuitive interface for decoration and customization. This paper illustrates an effective approach in Collaborative Commerce which can help enterprises face rapidly changing and unpredictable markets.

\section{B. Future Works}

WOA-based approaches have great potential to easily render systems interoperable, but innovations require extensive evaluation. Possible next steps include:

Step 1. Enhancing back-end cloud computing, to find the practical limitations of the WOA approach and to determine conditions which call for full SOA implementation;

Step 2. Developing Semantic Web search applications to increase the effectiveness of user searches for services and components;

Step 3. Developing interactive 3D models based on Web 2.0 concepts of sharing and reuse, to provide users with a more compelling and useable interface.

System security is another critical issue which must be addressed. While modern web technologies such as Ajax and mashup tools offer users a richer interface and experience, they also create security risks and vulnerabilities.

\section{REFERENCES}

[1] S. Singh, "Electronic Commerce Technologies Management," Encyclopedia of Multimedia Technology and Networking, pp. 278-282, 2005.

[2] E. Li, Y. Du, T. C. Wong, and W. Jacqueline, "Access control in collaborative commerce," Decision Support Systems, vol. 43, pp. 675-685, 2005. 
[3] D. Hinchcliffe. (February 2008). What is WOA? It's the future of service-oriented architecture (SOA). [Online]. Available: http://hinchcliffe.org/archive/2008/02/27/16617.aspx

[4] R. Smith. (July 2008). A simpler approach to SOA - Web-oriented architectures are easier to implement and offer a similar flexibility to SOA. [Online].

Available: http://www.informationweek.com/news/software/soa/showArticle.jht ml?articleID $=209904293$

[5] R. Battle and E. Benson, "Bridging the semantic web and web 2.0 with Representational State Transfer (REST)," Journal of Web Semantics: Science, Services and Agents on the World Wide Web, vol. 6, issue 1, pp. 61-69, February 2008.

[6] P. J. Muñoz-Merino, C. D. Kloos, and J. F. Naranjo, "Enabling interoperability for LMS educational services," Computer Standards \& Interfaces, vol. 31, issue 2, pp. 484-498, February 2009.

[7] UNSPSC. [Online]. Available: http://www.unspsc.org/

[8] Gartner. (2005). Whoa! We've got WOA. [Online]. Available: http://blog.gartner.com/blog/index.php?itemid $=400$

[9] R. Fielding, "Architectural Styles and the Design of Network-based Software Architectures," Ph.D. dissertation, Dept. Information and Computer Science, Univ. of California, Irvine. CA, USA, 2000.
[10] D. Hinchcliffe (April 2008). Web 2.0 success stories driving WOA and informing [Online]. Available: http://blogs.zdnet.com/Hinchcliffe/?p=168

[11] Ruby on Rails. [Online]. Available: http://rubyonrails.org/

[12] Restlet. [Online]. Available: http://www.restlet.org

[13] Cetia4. [Online]. Available: https://cetia4.dev.java.net/

[14] J. Yan, K. Ye, H. Wang, and Z. Hua, "Conceptual modeling of collaborative intelligent manufacturing for customized products: An ontological approach," in Proc. 2008 PACIS Conf., 2008, pp. 136-136.

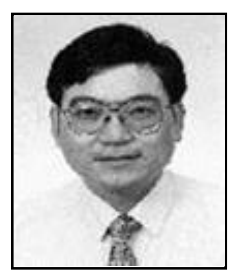

Chuan-Jun Su is an associate professor of the Industrial Engineering and Management Department at the Yuan Ze University, Taiwan. Prior to joining the University, he was an assistant professor with Hong Kong University of Science and Technology. His research interests include intelligent information systems, mobile agent technology, virtual reality, big data, and cloud computing. 